\title{
Characterization of clinically relevant copy-number variants from exomes of patients with inherited heart disease and unexplained sudden cardiac death
}

\author{
Emma S. Singer, BSc (Hons) ${ }^{1,2}$, Samantha B. Ross, BSc (Hons) ${ }^{1,2}$, Jon R. Skinner, MB, MD ${ }^{3,4}$, \\ Robert G. Weintraub, MBBS (Hons) ${ }^{5,6}$, Jodie Ingles, GradDipGenCouns, PhD ${ }^{1,2,7}$, \\ Christopher Semsarian, MB, PhD ${ }^{1,2,7}$ and Richard D. Bagnall, $\mathrm{PhD}$ (iD) ${ }^{1,2}$
}

Purpose: Copy-number variant (CNV) analysis is increasingly performed in genetic diagnostics. We leveraged recent gene curation efforts and technical standards for interpretation and reporting of $\mathrm{CNVs}$ to characterize clinically relevant $\mathrm{CNVs}$ in patients with inherited heart disease and sudden cardiac death.

Methods: Exome sequencing data were analyzed for CNVs using eXome-Hidden Markov Model tool in 48 established disease genes. $\mathrm{CNV}$ breakpoint junctions were characterized. CNVs were classified using the American College of Medical Genetics and Genomics technical standards.

Results: We identified eight CNVs in 690 unrelated probands (1.2\%). Characterization of breakpoint junctions revealed nonhomologous end joining was responsible for four deletions, whereas one duplication was caused by nonallelic homologous recombination between duplicated sequences in MYH6 and MYH7.
Identifying the precise breakpoint junctions determined the genomic involvement and proved useful for interpreting the clinical relevance of CNVs. Three large deletions involving TTN, MYBPC3, and $\mathrm{KCNH} 2$ were classified as pathogenic in three patients. Haplotype analysis of a deletion in ACTN2, found in two families, suggests the deletion was caused by an ancestral event.

Conclusion: CNVs infrequently cause inherited heart diseases and should be investigated when standard genetic testing does not reveal a genetic diagnosis.

Genetics in Medicine (2021) 23:86-93; https://doi.org/10.1038/s41436020-00970-5

Keywords: inherited heart disease; sudden cardiac death; copynumber variant; breakpoint junction; $M Y H 7$ duplication

\section{INTRODUCTION}

The inherited heart diseases include cardiomyopathies and arrhythmia syndromes, and they are an important cause of heart failure and sudden cardiac death in the young. ${ }^{1,2}$ Most inherited heart diseases display an autosomal dominant inheritance pattern and genetic testing of an affected proband is a clinical recommendation. ${ }^{3}$ Finding the precise genetic cause of disease can clarify an uncertain clinical diagnosis, may have implications for clinical management of the patient, and facilitates cascade genetic testing for early identification of at-risk family members.

For many families with inherited heart disease, standard genetic testing does not identify a pathogenic variant in the established disease genes. Alternative genetic causes that may have been missed include copy-number variants $(\mathrm{CNVs})^{4}$ and other structural rearrangements, such as translocations, ${ }^{5}$ and inversions. CNVs that involve the loss or gain of at least one exon can be detected with targeted gene panel or exome sequencing data by comparing the normalized sequencing read depth of exons between cases. ${ }^{6}$ Finding the precise ends of a CNV clarifies which genomic regions are involved, allows the design of a simple polymerase chain reaction (PCR)-based test for rapid genetic testing of at-risk family members, and can provide insights into the underlying mechanisms causing the $\mathrm{CNV}$.

Previous studies have reported CNVs in $0.3 \%$ to $11 \%$ of people with inherited heart disease, ${ }^{4}$ depending on the cohort studied and the criteria used to define a reportable CNV (Table S1). To improve the accuracy and consistency of interpreting the clinical relevance of CNVs, the American College of Medical Genetics and Genomics (ACMG) and the Clinical Genome Resource (ClinGen) recently introduced a semiquantitative, evidence-based scoring framework. ${ }^{8}$ It is not yet clear whether classifying CNVs using these new guidelines will alter the previously reported diagnostic yields.

In this study, we detected and validated CNVs found in evidence-based disease genes in patients with inherited heart disease and unexplained sudden cardiac death. We

${ }^{1}$ Agnes Ginges Centre for Molecular Cardiology at Centenary Institute, The University of Sydney, Sydney, NSW, Australia; ${ }^{2}$ Faculty of Medicine and Health, The University of Sydney, Sydney, NSW, Australia; ${ }^{3}$ Cardiac Inherited Disease Group New Zealand, Green Lane Paediatric and Congenital Cardiac Services, Starship Children's Hospital, Auckland, New Zealand; ${ }^{4}$ Department of Paediatrics Child and Youth Health, The University of Auckland, Auckland, New Zealand; ${ }^{5}$ Cardiology Department, Royal Children's Hospital, Melbourne, VIC, Australia; ${ }^{6}$ Murdoch Children's Research Institute and Department of Paediatrics, University of Melbourne, Melbourne, VIC, Australia; ${ }^{7}$ Department of Cardiology, Royal Prince Alfred Hospital, Sydney, NSW, Australia. Correspondence: Richard D. Bagnall (r.bagnall@centenary.org.au) 
characterized the breakpoint junctions to explore the underlying mechanisms causing the CNVs and used recent ACMG/ ClinGen guidelines to classify the clinical relevance.

\section{MATERIALS AND METHODS}

\section{Ethics statement}

Probands, or their next of kin, provided consent for genetic studies and the Sydney Local Health District Ethics Review Committee approved the study.

\section{Study cohort}

Probands diagnosed with a heritable cardiac disease were recruited from referrals to the Hypertrophic Cardiomyopathy and Genetic Heart Disease Clinics at Royal Prince Alfred Hospital, Sydney, Australia, and from the Royal Children's Hospital, Melbourne, Australia. Probands underwent clinical evaluations relevant to the presenting symptoms, such as electrocardiography, echocardiography, cardiac magnetic resonance imaging, and 24-hour Holter monitor, and a clinical diagnosis of a heritable cardiac disease was made in accordance with current guidelines. Pediatric patients with cardiomyopathy had metabolic, mitochondrial, and infectious causes excluded. Probands who died with a sudden cardiac death underwent a postmortem examination, including toxicological and histological analysis, as previously described. ${ }^{9}$

\section{Exome sequencing and read alignment}

DNA samples were extracted from fresh or frozen blood using the QIAamp DNA Mini Kit (Qiagen, Hilden, Germany) according to the manufacturer's instructions. Exome enrichment was performed using either the TruSeq (Illumina, California, USA) or SureSelect V4 plus UTR, or V5 all exon kits (Agilent Technologies, California, USA) depending on the most recent kit available during the study period. Exome sequencing was performed as previously described. ${ }^{10}$

\section{Detection of CNVs from exome sequencing read depth}

Exome read alignments were used to detect CNVs in batches according to the exome enrichment kit. We detected CNVs using eXome-Hidden Markov Model (XHMM) ${ }^{6}$ software, in strict accordance with a detailed protocol, ${ }^{11}$ which uses the normalized sequencing read depth of exons to determine the presence of a CNV against a background of pooled samples. XHMM calculates a probability that a CNV event has occurred at a specified region (Q-some score) and CNVs with a Q-some score $<55$ were excluded. We looked for CNVs that overlap with 43 established cardiac disease genes plus 5 genes that cause disease with cardiac involvement (Table S2).

\section{Quantitative PCR}

Quantitative PCR (qPCR) was performed with primers designed using primer3 plus (http://www.bioinformatics.nl/ cgi-bin/primer3plus/primer3plus.cgi). Primer pairs had a melting temperature between $57^{\circ} \mathrm{C}$ and $63^{\circ} \mathrm{C}$ and amplified products between 100 and 350 base pairs (bp) (Table S3).
qPCR of patient and three control DNA samples was performed in triplicate in reactions containing $2 \mathrm{ng} / \mu \mathrm{l} \mathrm{DNA}$, $6 \mu \mathrm{l}$ Fast SYBR green (Applied Biosystems, California, USA), and $300 \mathrm{nM}$ of each primer in a final volume of $10 \mu \mathrm{l}$. Amplification efficiencies were determined from a standard curve using the MxPro-Mx3005P v4.10 software (Agilent Technologies), with efficiencies between $90 \%$ and $110 \%$ deemed usable. Optimized qPCR assays were performed on the Stratagene Mx3005P platform (Agilent Technologies) with an initial activation at $50^{\circ} \mathrm{C}$ for 2 minutes, followed by $95^{\circ} \mathrm{C}$ for 2 minutes, and then 40 cycles of $95^{\circ} \mathrm{C}$ for 15 seconds and 60 seconds at the optimized annealing and extension temperature, with a final single dissociation from $60^{\circ} \mathrm{C}$ to $95^{\circ} \mathrm{C}$. Ct values were corrected for primer efficiency and normalized to a PCR product of GAPDH. Relative quantification of DNA was determined using the Pfaffl method. ${ }^{12}$

\section{PCR and Sanger sequencing}

PCR amplifications to confirm CNVs were performed using primer pairs that flanked a deletion or duplication breakpoint junction, plus a third primer that amplifies the normal chromosome as a positive control (Table S3). PCR and Sanger sequencing were performed as previously described. ${ }^{10}$

\section{Haplotype analysis}

Haplotype analysis of five polymorphic variable number of tandem repeat loci within and flanking ACTN2 was performed as described previously. ${ }^{13}$

\section{RESULTS}

\section{Cohort demographics and clinical characteristics}

We included 690 probands diagnosed with an inherited heart disease or unexplained sudden cardiac death who had undergone exome sequencing. The average age was 37 years ( \pm 18.9 years), the majority were males, $(n=439 ; 63 \%), 28 \%$ had a family history of disease, and $33 \%$ had a family history of sudden cardiac death. The most common diagnosis was hypertrophic cardiomyopathy $(n=317 ; 46 \%)$ followed by unexplained sudden cardiac death $(n=146 ; 21 \%)$, dilated cardiomyopathy ( $n=86 ; 12 \%)$, left ventricular noncompaction $(n=57 ; 8 \%)$, Brugada syndrome $(n=36 ; 5 \%)$, long QT syndrome $(n=18 ; 3 \%)$, arrhythmogenic cardiomyopathy $(n=13 ; 2 \%)$, restrictive cardiomyopathy $(n=11 ; 2 \%)$, and catecholaminergic polymorphic ventricular tachycardia $(n=$ 6; 1\%) (Table 1).

\section{Detection of CNVs from exome sequencing read depth}

We detected $8 \mathrm{CNVs}$ in 8 probands (1.2\%) in a panel of 48 genes with established cardiac disease involvement (Table 2; Fig. S1). The CNVs comprise 5 deletions and 3 duplications with a predicted size range of $14 \mathrm{~kb}$ to $795 \mathrm{~kb}$. We detected a 14-kb deletion spanning part of $M Y B P C 3$, and a tandem duplication of part of $M Y H 7$ and MYH6, in two people with hypertrophic cardiomyopathy and no alternate genetic diagnosis. We detected a 795-kb deletion spanning 26 genes, including $\mathrm{KCNH} 2$, in a person with unexplained sudden 
Table 1 Cohort demographics and clinical characteristics.

\begin{tabular}{|c|c|c|c|c|c|c|}
\hline Diagnosis & $\begin{array}{l}\text { Number of } \\
\text { probands }\end{array}$ & Sex & $\begin{array}{l}\text { Average age at } \\
\text { diagnosis (years) }\end{array}$ & $\begin{array}{l}\text { Family history } \\
\text { of disease }\end{array}$ & $\begin{array}{l}\text { Family history of sudden } \\
\text { cardiac death }\end{array}$ & $\begin{array}{l}\text { Genetic testing } \\
\text { diagnostic yield }\end{array}$ \\
\hline $\mathrm{HCM}$ & 317 & $\begin{array}{l}\text { Male } 214 \\
(67.5 \%)\end{array}$ & $45.2( \pm 17.4)$ range $1-88$ & $75 / 302(24.8 \%)$ & $38 / 308(12.3 \%)$ & $36 \%$ \\
\hline SUD & 146 & $\begin{array}{l}\text { Male } 103 \\
(70.5 \%)\end{array}$ & $23.7( \pm 11.7)$ range $1-66$ & Unknown & $146 / 146(100 \%)$ & $21 \%$ \\
\hline DCM & 86 & $\begin{array}{l}\text { Male } 44 \\
(51.2 \%)\end{array}$ & $40( \pm 19.8)$ range $0-69$ & $38 / 80(47.5 \%)$ & $8 / 76(10.5 \%)$ & $29 \%$ \\
\hline LVNC & 57 & $\begin{array}{l}\text { Male } 27 \\
(47.4 \%)\end{array}$ & $35.2( \pm 19.3)$ range $0-77$ & $18 / 55(32.7 \%)$ & 9/53 (16.9\%) & $23 \%$ \\
\hline $\mathrm{BrS}$ & 36 & $\begin{array}{l}\text { Male } 33 \\
(91.7 \%)\end{array}$ & $42.6( \pm 12.6)$ range $19-60$ & $6 / 36(16.7 \%)$ & $4 / 36(11.1 \%)$ & $14 \%$ \\
\hline LQTS & 18 & $\begin{array}{l}\text { Male } 3 \\
(16.7 \%)\end{array}$ & $28.3( \pm 22.3)$ range $0-42$ & $5 / 17(29.4 \%)$ & $5 / 18(27.8 \%)$ & $36 \%$ \\
\hline ARVC & 13 & $\begin{array}{l}\text { Male } 7 \\
(53.8 \%)\end{array}$ & $41.1( \pm 19.5)$ range $15-64$ & $0 / 11(0 \%)$ & $3 / 11(27.3 \%)$ & $8 \%$ \\
\hline RCM & 11 & $\begin{array}{l}\text { Male } 5 \\
(45.5 \%)\end{array}$ & $4.2( \pm 3.7)$ range $0-9$ & $3 / 10(30 \%)$ & $1 / 10(10 \%)$ & $64 \%$ \\
\hline CPVT & 6 & $\begin{array}{l}\text { Male } \\
3(50 \%)\end{array}$ & $28.3( \pm 15.8)$ range $6-40$ & $2 / 6(33.3 \%)$ & $2 / 6(33.3 \%)$ & $17 \%$ \\
\hline
\end{tabular}

CPVT catecholaminergic polymorphic ventricular tachycardia, DCM dilated cardiomyopathy, HCM hypertrophic cardiomyopathy, LQTS long QT syndrome, LVNC left ventricular noncompaction, RCM restrictive cardiomyopathy, SUD unexplained sudden cardiac death.

Table 2 CNVs detected in 48 established cardiac disease genes.

\begin{tabular}{lllllll} 
Patient ID & Diagnosis & CNV class & Predicted CNV interval (hg19) & CNV length (kb) & Quality score & Most clinically relevant gene \\
\hline CMD1 & HCM & Deletion & chr11:47345261-47359431 & 14.2 & 93 & MYBPC3 \\
NSW76A & SUD & Deletion & chr7:149981783-150777008 & 795.2 & 93 & KCNH2 \\
CBD1 & DCM & Deletion & chr2:179417075-179501545 & 84.5 & 99 & TTN \\
CIJ3 & DCM & Deletion & chr10:68280379-68535287 & 254.9 & 92 & CTNNA3 \\
AHM1 & LVNC & Deletion & chr1:236882093-236897871 & 15.8 & 76 & ACTN2 \\
AYQ1 & HCM & Duplication & chr14:23859337-23889071 & 29.7 & 95 & MYH7 \\
CBT1 & LVNC & Duplication & chr10:112540513-112595730 & 55.2 & 92 & RBM20 \\
BLX1 & LQTS & Duplication & chr6:118832413-118953777 & 121.4 & 94 & $P L N$ \\
\hline
\end{tabular}

CNV copy-number variant, DCM dilated cardiomyopathy, HCM hypertrophic cardiomyopathy, LQTS long QT syndrome, LVNC left ventricular noncompaction, SUD unexplained sudden cardiac death.

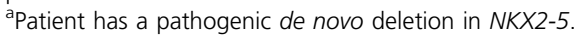

cardiac death and no alternate genetic diagnosis. In two people with dilated cardiomyopathy and no alternate genetic diagnosis, we detected an 84-kb deletion involving 110 exons encoding part of the titin A band, and a $255-\mathrm{kb}$ intragenic deletion of CTNNA3. In two people with left ventricular noncompaction we detected a $16-\mathrm{kb}$ intragenic deletion of $A C T N 2$, and a $55-\mathrm{kb}$ intragenic duplication of RBM20 in a person who has a de novo pathogenic frameshift variant in NKX2-5. Finally, we detected a 121-kb duplication including the entire $P L N$ gene in a person with long QT syndrome who did not have an alternate genetic diagnosis for this disease.

\section{Breakpoint junctions characterized from exome sequencing reads}

The precise breakpoint junctions of three deletions were found within exome sequencing reads (Fig. 1). The breakpoints of a deletion involving $M Y B P C 3$ were found within a cluster of reads that aligned to an AluSx repeat within intron 32 of $M A D D$ and intron 23 of MYBPC3. Sanger sequencing of a PCR product amplified across this junction in the proband's DNA confirmed a deletion of $19 \mathrm{~kb}$ (NC_000011.9: g.47340871_47359757del). There were four nucleotides of homology at the ends of the breakpoint in the reference sequence (Fig. 1a). The breakpoint of an intragenic deletion in TTN was found within a cluster of reads that aligned to intron 224 and exon 335 of the major cardiac isoform of TTN (NM_001256850.1). Sanger sequencing of a PCR product amplified across this junction in the proband, and the absence of this product in his parent's DNA, confirmed a de novo deletion of $86 \mathrm{~kb}$ (NC_000002.11:g.179418282_179504054del). There were four nucleotides of homology at the ends of the breakpoint in the reference sequence (Fig. 1b). Finally, the 

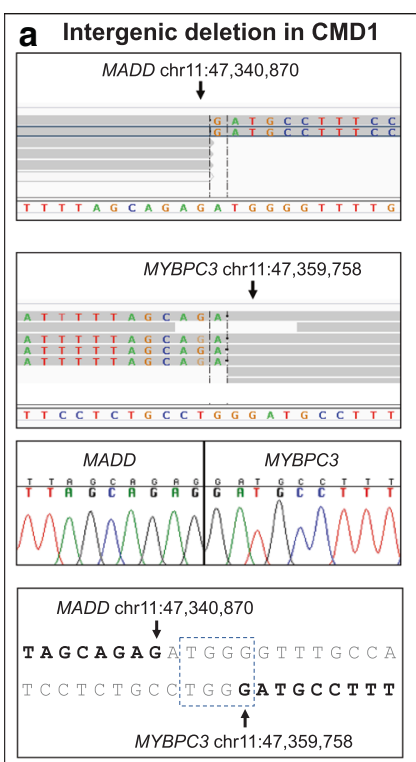
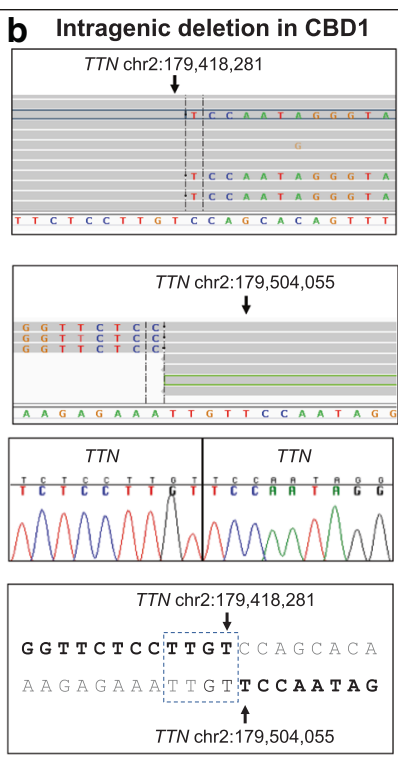
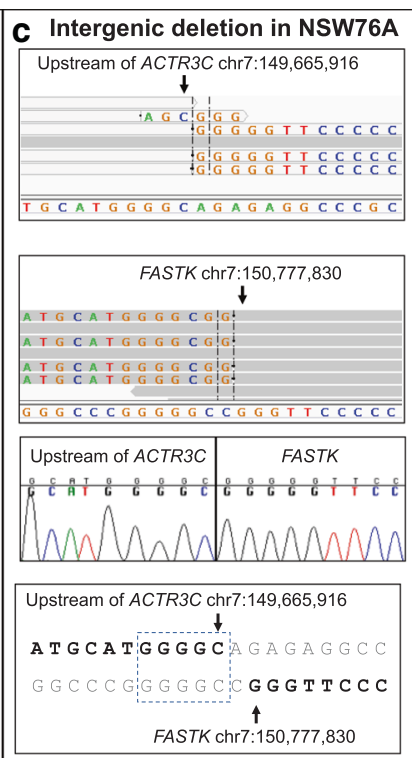

Fig. 1 Deletion junctions characterized from exome sequencing split reads. Characterization of deletion breakpoints within (a) intron 32 of $M A D D$ and intron 23 of MYBPC3 in patient CMD1; (b) intron 224 and exon 335 of TTN in patient CBD1; and (c) a 1.1 Mb deletion involving KCNH2 in patient NSW76A. Top two panels show sequence reads spanning breakpoint junctions in the reference sequence. Gray horizontal bars are sequencing reads; nucleotides that differ from the reference sequence are shown in colors. Middle panels show Sanger sequencing electropherograms across the breakpoint junctions. Bottom panels show alignments of breakpoint junction sequences (bold) in the reference sequence with regions of microhomology in stippled boxes.

breakpoint junction of a large deletion involving 26 genes, including $\mathrm{KCNH} 2$, was found in a cluster of reads aligning $\sim 300 \mathrm{~kb}$ upstream of the $A C T R 3 C$ gene and to exon 1 of FASTK. Sanger sequencing of a PCR product amplified across this junction in the proband's DNA confirmed a deletion of $1.1 \mathrm{Mb}$ (NC_000007.13:g.149665917_150777829del). There were five nucleotides of homology at the ends of the breakpoint in the reference sequence (Fig. 1c). The breakpoints of the remaining five CNVs could not be found within the exome sequencing read alignments.

\section{Breakpoint junctions characterized using $\mathrm{QPCR}$ and PCR}

In a female with left ventricular noncompaction, qPCR confirmed a deletion of exons 3 to 6 in ACTN2 (Fig. 2a). Sanger sequencing of a PCR product with primers annealing in the flanking introns confirmed a 9.3-kb intragenic deletion from intron 2 to exon 6 of ACTN2 (NC_000001.10: g.236881685_236891006del), and there were three nucleotides of homology at the ends of the breakpoints in the reference sequence (Fig. 2a). We used the same primer pair to amplify and Sanger verify an identical breakpoint junction in two affected members of an apparently unrelated family (family CKE) with hypertrophic cardiomyopathy, in which a deletion involving ACTN2 exons 3 to 6 was previously detected using multiplex ligation-dependent probe amplification. Genotyping of five polymorphic dinucleotide repeats surrounding the ACTN2 locus showed that both families share a common haplotype containing the deletion; thus, the CNV in both families was likely caused by the same ancestral deletion event (Fig. S2). We suspected a duplication involving MYH6 and MYH7 might be caused by homologous recombination between misaligned copies of a 486-bp segment with $99.4 \%$ sequence identity in exon 26 of MYH6 and exon 27 of MYH7 (Fig. S3), as previously proposed for a similar duplication in a family with hypertrophic cardiomyopathy. ${ }^{14}$ qPCR verified the duplication and Sanger sequencing of a 1.1-kb PCR product amplified using primers annealing to intron 25 of MYH6 and exon 28 of MYH7 confirmed that the tandem duplication was indeed caused by this mechanism (Fig. 2b).

\section{CNVs with uncharacterized breakpoint junctions}

Duplications involving RBM20 and PLN were confirmed using $\mathrm{qPCR}$ only (Fig. S4), whereas an intragenic deletion involving exons 8 to 11 of CTNNA3 was not further assessed because similar deletions in this region are common in the general population. The RBM20 duplication spans exons 2 to 14 with the breakpoints lying within the 136-kb intron 1 of $R B M 20$ and the $32 \mathrm{~kb}$ of intergenic space between RBM20 and the adjacent PDCD4 gene (Fig. S4a). The breakpoints of a duplication of exons 2 to 5 of CEP85L, which includes the whole of $P L N$, fall within the $18 \mathrm{~kb}$ of intron 1 and the $19 \mathrm{~kb}$ of intron 5 of CEP85L (Fig. S4b). The breakpoints of these $\mathrm{CNV}$ lay within very large introns or intergenic regions and could not be further localized despite repeated attempts of long-range PCR using various primer combinations.

\section{Classifying the pathogenicity of CNVs using the ACMG/ ClinGen framework}

We classified the eight CNVs using the recent ACMG/ GlinGen framework for the interpretation and reporting of CNVs (Table 3). Three deletions, involving MYBPC3, $\mathrm{KCNH} 2$, and TTN, were classified as pathogenic, one deletion 


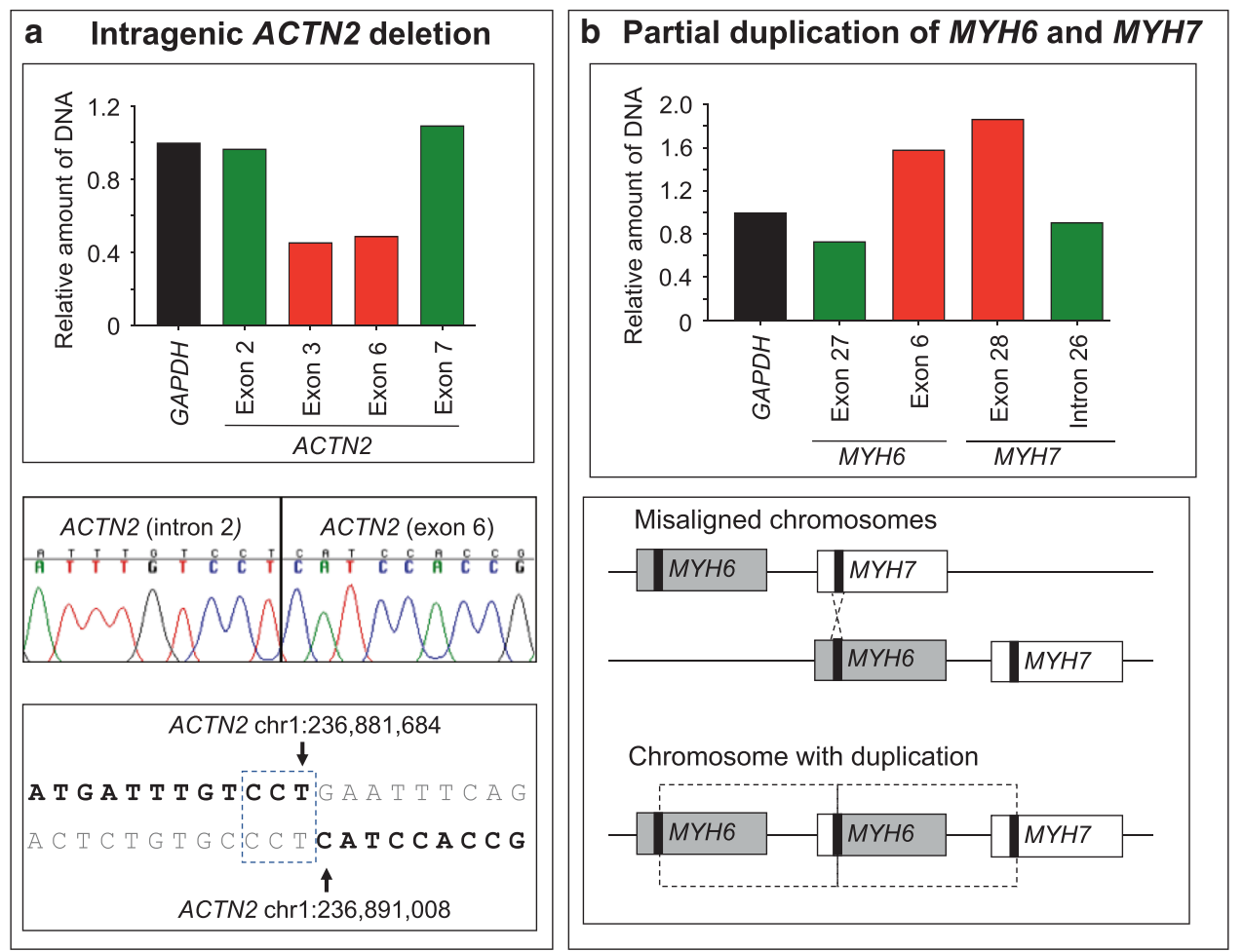

Fig. 2 Copy-number variants (CNVs) characterized with quantitative polymerase chain reaction (qPCR) and PCR amplification. Upper panels show qPCR of segments within and flanking (a) an ACTN2 deletion in patient AHM1 and (b) a partial duplication of MYH6 and MYH7 in patient AYQ1. Relative DNA copy number is shown for an exon of GAPDH (black bars), exons flanking the CNVs (green bars) and exons within the CNVs (red bars). Sanger sequencing electropherograms across the ACTN2 deletion breakpoint (a, central panel) and alignments of breakpoint junctions in the reference sequence (a, lower panel) are shown with region of microhomology in stippled boxes. Proposed mechanism of nonallelic homologous recombination between misaligned duplicated segment of MYH6 and MYH7 (black boxes), with resulting duplicated segment in stippled box (b, lower panel).

within ACTN2 and three duplications involving $M Y H 7$, $R B M 20$, and $P L N$ were classified as variants of uncertain significance (VUS), and an CTNNA3 intragenic deletion was classified as benign. The deletion involving MYBPC3 removes the last 12 exons of this gene, predicting a loss of function, and was found in a male diagnosed with hypertrophic cardiomyopathy at age 43 years. He has no known family history of disease and parental DNA was not available for cascade genetic testing. A very large deletion that removes 26 genes, including $\mathrm{KCNH} 2$, was found in a female who died during sleep at age 17 years. None of the other deleted genes have cardiac disease involvement (Table S4). She had no significant medical history prior to death, her postmortem investigation did not reveal a cause of death, there was no family history of cardiac disease, and parental DNA was not available. Haploinsufficiency of $\mathrm{KCNH} 2$ causes the long QT syndrome, which is associated with an increased risk of unexplained sudden cardiac death. The de novo deletion within TTN, which removes part of the A band domain and causes a loss of function, was found in a boy diagnosed with dilated cardiomyopathy at birth. He has since undergone heart transplantation. The frameshifting deletion of exons 3 to 6 of ACTN2 was found in a female with left ventricular noncompaction and classified as a VUS. Her twin brother has bicuspid aortic valve and a nephew has had aortic stenosis since birth and has undergone aortic valve replacement. No
DNA was available for cascade genetic testing. A partial tandem duplication of MYH7 and MYH6 classified as VUS in a male with hypertrophic cardiomyopathy despite a similar duplication segregating with seven affected members of a family with hypertrophic cardiomyopathy. ${ }^{14}$ Two duplications involving RBM20 and PLN were classified as VUS as triplosensitivity has not been established in these genes and the duplications would leave both copies of the genes intact. The RBM20 duplication was found in a female aged 40 years who has a noncompacted to compacted myocardial thickness ratio of up to $3.4: 1$ on cardiac magnetic resonance image (MRI). She had an atrial septal defect closure at age 4 years and her twin sister, presumed identical, died suddenly at age 19 years with no cause identified at postmortem examination. They both harbor a de novo nonsense variant in NKX2-5. The PLN duplication was found in a female aged 30 years with LQTS. She has had recurrent syncope since childhood and her QTc measured $470 \mathrm{~ms}$ on electrocardiogram (ECG) and $490 \mathrm{~ms}$ on Holter monitor. Her father and paternal grandfather had an unexplained sudden death at ages 39 and 38 years, respectively, and a cousin died during sleep at age 10 years. No DNA is available. Her son has a QTc in the upper limits of normal, measuring $445 \mathrm{~ms}$ at age 5 years. The deletion within CTNNA3 was classified as benign as this region is also deleted in $145 / 10,847$ (1.3\%) people in the general population. ${ }^{15}$ 


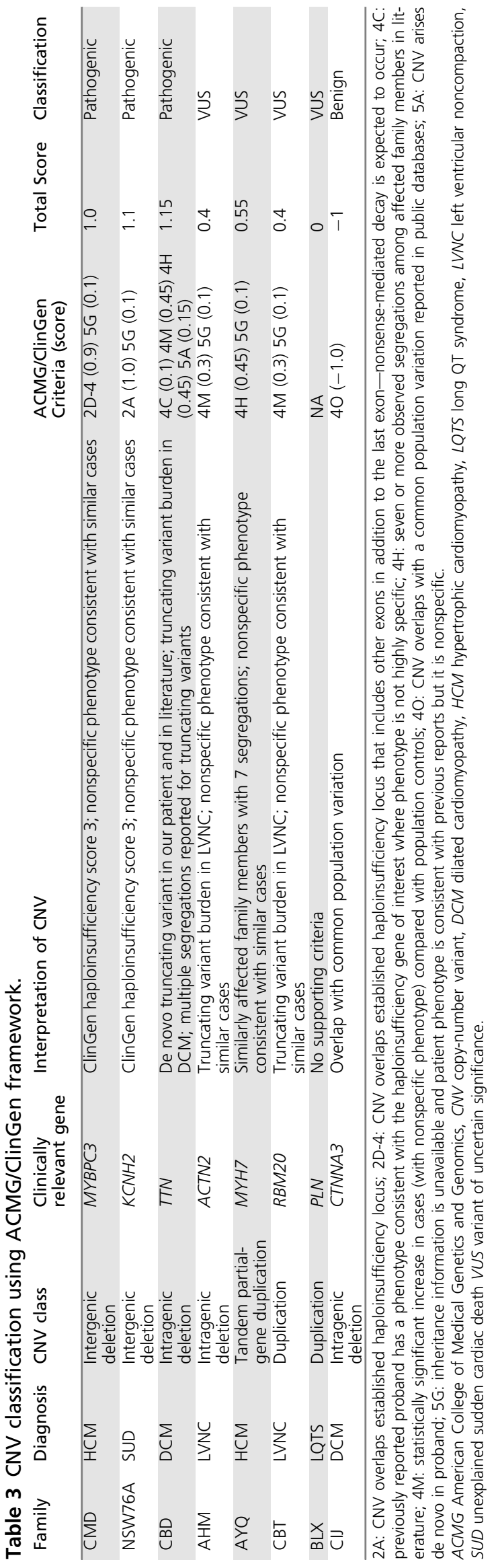

\section{DISCUSSION}

We performed CNV analysis using exome sequencing data of 690 unrelated probands diagnosed with an inherited heart disease or with sudden cardiac death and found eight CNVs in established disease genes in eight probands (1.2\%). We mapped the breakpoints of four deletions and one duplication using a combination of exome sequencing read analysis, qPCR, and PCR, and confirmed the presence of two duplications using qPCR. Four deletions likely arose via nonhomologous end joining and one duplication is the result of nonallelic homologous recombination between misaligned copies of a duplicated sequence in MYH6 and MYH7. Using ACMG/ClinGen technical standards for interpretation of $\mathrm{CNVs}$, we classified three deletions as pathogenic giving an overall yield of clinically relevant CNVs of $0.4 \%$. Our results highlight that clinically relevant $\mathrm{CNV}$ s are a rare cause of inherited heart disease, but can be readily detected using available sequencing data. We recommend that existing sequencing data should be investigated for CNVs as a secondary analysis step in people with inherited heart disease or sudden cardiac death who have an otherwise indeterminate genetic test.

\section{Factors influencing the yield of CNVs}

The yield of CNVs in our study was influenced by restricting our analysis to 48 established cardiac disease genes. As with standard genetic testing, inclusion of additional cardiac expressed genes that currently lack sufficient evidence for disease causality would have increased our yield of VUS, with minimal or no increase in pathogenic or likely pathogenic CNVs. For instance, we characterized an intragenic deletion of MYPN and an inverted duplication of MYLK3 in a person with hypertrophic cardiomyopathy and dilated cardiomyopathy, respectively (Fig. S5). These were not considered further as the association with cardiac disease for these two genes is currently limited. ${ }^{16,17}$ Our yield of CNVs was also influenced by screening an unselected cohort of patients, rather than focusing on patients who had a prior indeterminate genetic test, which would enrich for CNVs that are usually missed with standard genetic testing. When considering disease subgroups, we found a clinically relevant $\mathrm{CNV}$ in 1/317 hypertrophic cardiomyopathy, 1/146 unexplained sudden cardiac death, and 1/86 dilated cardiomyopathy. Our study is likely underpowered to identify clinically relevant CNVs in other disease subgroups, including long QT syndrome, where CNVs in KCNQ1, KCNH2, and SCN5A have been reported in up to $11 \%$ of cases who have an otherwise indeterminate genetic test. ${ }^{18}$ Although several tools can detect structural variants from exome sequencing data, we used XHMM as it focuses on finding rare CNVs and filters outliers in regions with high GC content and low complexity regions. XHMM has been used to detect CNVs in cohorts of up to 60,000 individuals, ${ }^{19}$ uses all available samples for data normalization, and has a low false positive rate based on comparison studies. ${ }^{20}$ XHMM has lower accuracy with detecting common or complex CNVs, or single-exon CNVs, 
and using a combination of $\mathrm{CNV}$ callers may further increase the diagnostic yield.

\section{Mapping breakpoint junctions}

A key step in the ACMG/ClinGen guidelines for reporting of CNVs involves assessing the genomic content of CNVs, and this was aided by characterizing the breakpoint junctions. This determined which exons were involved in the CNVs and revealed that some CNVs included additional exons to those predicted by XHMM software. It also clarified whether deletions disrupted the protein reading frame and whether duplications occurred in tandem or represented rare insertional translocations elsewhere in the genome, which would leave the duplicated gene intact. The quickest approach to locating breakpoint junctions was finding exome sequencing reads that mapped across the breakpoint junction, but this was only possible if at least one end of the CNV fell within a sequenced region. PCR was also a straightforward approach to mapping breakpoint junctions, but was only successful when the ends of the CNV fell within relatively short intervals. Mapping the precise breakpoints of one duplication and one deletion was not successful as the breakpoints fell within very large intervals. Further narrowing of these intervals would require additional testing, such as qPCR of consecutive regions along the long intervals, or chromosomal microarray. Despite these shortcomings, we have shown how analysis of available exome sequencing data can be a costeffective approach to characterize CNVs and pinpoint the breakpoint junction.

\section{Molecular mechanisms causing CNVs}

Our CNVs were caused by rare events that are unlikely to be recurrent. Four deletions likely arose through nonhomologous end joining events at sites of microhomology. This is consistent with previous reports in which the ends of deletions involving $K C N H 2, K C N Q 1$, and $M Y B P C 3$ have been mapped to different loci that show stretches of microhomology, or reside within Alu repeat sequences. ${ }^{21}$ In our cohort, only one end of one deletion occurred within an Alu repeat, found within intron 32 of $M A D D$. Alu repeats are often found at the ends of CNVs and it has been suggested these sequences may be prone to recombination, ${ }^{22}$ or are often found at the ends of CNVs simply because Alu repeats make up almost $11 \%$ of the human genome. ${ }^{23}$ In contrast to the unique deletion events, we found a partial duplication of MYH7 and MYH6 that was caused by nonallelic homologous recombination between misaligned copies of a homologous sequence found in these tandemly arranged genes. This duplication may be a low frequency recurrent event as it has been previously reported in a family with hypertrophic cardiomyopathy. ${ }^{14}$ The duplication leaves the flanking copies of MYH6 and MYH7 intact and creates a novel hybrid $\mathrm{MYH6/}$ MYH7 gene, which would be easily missed using standard genetic testing approaches. The reciprocal outcome would be a chromosome in which the flanking $M Y H 6$ and $M Y H 7$ genes are replaced by a hybrid MYH6/MYH7 gene. While, to our knowledge, this derivative chromosome has not yet been observed, we postulate that such a chromosomal arrangement would also be found in association with hypertrophic cardiomyopathy.

\section{Clinical classification of CNVs using ACMG/ClinGen standards}

We classified as pathogenic three deletions in genes with clear evidence for haploinsufficiency, and these deletions either arose de novo (TTN) or the patient's phenotype was consistent with what had been described in similar cases (KCNH2, MYBPC3). These three patients had undergone prior genetic testing with no cause found and highlight the importance of $\mathrm{CNV}$ analysis in providing a genetic diagnosis. A further two CNVs had supportive evidence for disease involvement but only classified as VUS using the current ACMG/ClinGen guidelines. A deletion of exons 3 to 6 of ACTN2, encoding part of a calponin homology domain and predicting a loss of reading frame, was found in a female with left ventricular noncompaction. ACTN2 is awaiting adjudication as a haploinsufficiency gene; however, the gene is constrained for loss-of-function variants and there is an overrepresentation of loss-of-function variants in people with left ventricular noncompaction when compared with controls (unpublished data). If ACTN2 is adjudicated as a haploinsufficiency gene, the intragenic deletion would classify as pathogenic using the PVS1 rule, which evaluates null variants in genes for which loss of function is a known disease mechanism. Surprisingly, we also found the same deletion segregating in two affected members of a family with hypertrophic cardiomyopathy, and both families share the deletion on the same haplotype, suggesting a common origin. Although both families have different clinical presentations, we have previously described a pathogenic Ala119Thr missense variant in ACTN2 segregating with hypertrophic cardiomyopathy, left ventricular noncompaction and sudden cardiac death. ${ }^{13,24}$ Another CNV with supportive evidence for disease involvement, but which only classified as VUS, was a partial tandem duplication of MYH7 and MYH6, found in a male with hypertrophic cardiomyopathy. The same CNV was previously shown to segregate in seven affected members of a family with hypertrophic cardiomyopathy. ${ }^{14}$ Although the duplication will leave the MYH7 and MYH6 genes intact, it is predicted to create a novel hybrid gene encoding the first 1244 amino acids of MYH6 followed by the last 693 amino acids of MYH7 under the influence of the MYH6 promoter. This hybrid gene may cause hypertrophic cardiomyopathy by incorporation of a "poison peptide" in the sarcomere that impairs contractile function. The creation of a novel hybrid protein is not currently adjudicated in the AGMG criteria, although such fusion proteins underlie various cancers. While the ACMG/ClinGen technical standard for interpretation of CNVs will likely reduce inconsistent classifications across laboratories, the stringent criteria needed to achieve a likely pathogenic or pathogenic classification may reduce the currently reported yield of clinically relevant $\mathrm{CNVs}$ for a 
number of diseases. Adherence to the guidelines is voluntary and professional judgment may be required to interpret the relevance of very rare $\mathrm{CNV}$ events that are unlikely to reoccur in other families.

\section{Conclusion}

CNVs are a rare cause of inherited heart disease and sudden cardiac death, but can be detected with analysis of available exome sequencing data. Finding a clinically relevant $\mathrm{CNV}$ provides a genetic diagnosis to aid clinical management of the family, which would otherwise not be possible. We therefore reiterate that $\mathrm{CNV}$ analysis should be performed as a secondary analysis when no genetic cause of disease is found with standard genetic testing. Within the established inherited heart disease genes, we found most CNVs arise via rare, often family specific events, with the exception of a duplication involving part of the MYH6 and MYH7 genes, which may be a low-level recurrent event. Finding the breakpoint junctions is an important step in characterization of CNVs and helps with the interpretation using the recent ACMG/ClinGen technical standards for reporting of CNVs. Future interpretation of rare CNVs will be facilitated by sharing case examples.

\section{SUPPLEMENTARY INFORMATION}

The online version of this article (https://doi.org/10.1038/s41436020-00970-5) contains supplementary material, which is available to authorized users.

\section{ACKNOWLEDGEMENTS}

C.S. is the recipient of a National Health and Medical Research Council (NHMRC) Practitioner Fellowship (1154992); J.I. is the recipient of an NHMRC Career Development Fellowship (1162929); R.D.B. is the recipient of a New South Wales Health Cardiovascular Disease Senior Scientist Grant.

\section{DISCLOSURE}

J.I. receives research grant support from Myokardia, Inc. The other authors declare no conflicts of interest.

Publisher's note Springer Nature remains neutral with regard to jurisdictional claims in published maps and institutional affiliations.

\section{REFERENCES}

1. Al-Khatib SM, Stevenson WG, Ackerman MJ, et al. AHAVACC/HRS guideline for management of patients with ventricular arrhythmias and the prevention of sudden cardiac death: executive summary: a report of the American College of Cardiology/American Heart Association Task Force on Clinical Practice Guidelines and the Heart Rhythm Society. Circulation. 2018;138:e210-e271.

2. McKenna WJ, Maron BJ, Thiene G. Classification, epidemiology, and global burden of cardiomyopathies. Circ Res. 2017;121:722-730.
3. Ackerman MJ, Priori SG, Willems S, et al. HRS/EHRA expert consensus statement on the state of genetic testing for the channelopathies and cardiomyopathies: this document was developed as a partnership between the Heart Rhythm Society (HRS) and the European Heart Rhythm Association (EHRA). EP Europace. 2011;13:1077-1109.

4. Mates J, Mademont-Soler I, Fernandez-Falgueras A, et al. Sudden cardiac death and copy number variants: what do we know after 10 years of genetic analysis? Forensic Sci Int Genet. 2020;47:102281.

5. Yeates L, Ingles J, Gray B, et al. A balanced translocation disrupting SCN5A in a family with Brugada syndrome and sudden cardiac death. Heart Rhythm. 2019;16:231-238.

6. Fromer M, Moran JL, Chambert $\mathrm{K}$, et al. Discovery and statistical genotyping of copy-number variation from whole-exome sequencing depth. Am J Hum Genet. 2012;91:597-607.

7. Liu P, Carvalho CMB, Hastings PJ, Lupski JR. Mechanisms for recurrent and complex human genomic rearrangements. Curr Opin Genet Dev. 2012;22:211-220.

8. Riggs ER, Andersen EF, Cherry AM, et al. Technical standards for the interpretation and reporting of constitutional copy-number variants: a joint consensus recommendation of the American College of Medical Genetics and Genomics (ACMG) and the Clinical Genome Resource (ClinGen). Genet Med. 2020;22:245-257.

9. Bagnall RD, Weintraub RG, Ingles J, et al. A prospective study of sudden cardiac death among children and young adults. N Engl J Med. 2016:374:2441-2452.

10. Bagnall RD, Ingles J, Yeates L, Berkovic SF, Semsarian C. Exome sequencing-based molecular autopsy of formalin-fixed paraffinembedded tissue after sudden death. Genet Med. 2017:19:1127-1133.

11. Fromer M, Purcell SM. Using XHMM software to detect copy number variation in whole-exome sequencing data. Curr Protoc Hum Genet. 2014;81:7.23.1-7.23.21.

12. Pfaffl MW. A new mathematical model for relative quantification in realtime RT-PCR. Nucleic Acids Res. 2001;29:e45.

13. Bagnall RD, Molloy LK, Kalman JM, Semsarian C. Exome sequencing identifies a mutation in the ACTN2 gene in a family with idiopathic ventricular fibrillation, left ventricular noncompaction, and sudden death. BMC Med Genet. 2014;15:99.

14. Tanigawa G, Jarcho JA, Kass $S$, et al. A molecular basis for familial hypertrophic cardiomyopathy: an alpha/beta cardiac myosin heavy chain hybrid gene. Cell. 1990;62:991-998.

15. Collins RL, Brand $\mathrm{H}$, Karczewski KJ, et al. A structural variation reference for medical and population genetics. Nature. 2020;581:444-451.

16. Purevjav E, Arimura T, Augustin S, et al. Molecular basis for clinical heterogeneity in inherited cardiomyopathies due to myopalladin mutations. Hum Mol Genet. 2012;21:2039-2053.

17. Tobita T, Nomura S, Morita H, et al. Identification of MYLK3 mutations in familial dilated cardiomyopathy. Sci Rep. 2017;7:17495.

18. Eddy CA, MacCormick JM, Chung SK, et al. Identification of large gene deletions and duplications in KCNQ1 and $\mathrm{KCNH} 2$ in patients with long QT syndrome. Heart Rhythm. 2008;5:1275-1281.

19. Ruderfer DM, Hamamsy T, Lek $M$, et al. Patterns of genic intolerance of rare copy number variation in 59,898 human exomes. Nat Genet. 2016;48:1107-1111.

20. Tan R, Wang Y, Kleinstein SE, et al. An evaluation of copy number variation detection tools from whole-exome sequencing data. Hum Mutat. 2014;35:899-907.

21. Chanavat V, Seronde MF, Bouvagnet P, Chevalier P, Rousson R, Millat G. Molecular characterization of a large MYBPC3 rearrangement in a cohort of 100 unrelated patients with hypertrophic cardiomyopathy. Eur J Med Genet. 2012;55:163-166.

22. Witherspoon DJ, Watkins WS, Zhang $Y$, et al. Alu repeats increase local recombination rates. BMC Genomics. 2009;10:530.

23. Lander ES, Linton LM, Birren B, et al. Initial sequencing and analysis of the human genome. Nature. 2001;409:860-921.

24. Chiu C, Bagnall RD, Ingles J, et al. Mutations in alpha-actinin-2 cause hypertrophic cardiomyopathy: a genome-wide analysis. J Am Coll Cardiol. 2010;55:1127-1135 